\title{
A novel classification system for the evaluation and reconstruction of oral defects following oncological surgery
}

\author{
WEI WEI LIU, CHU YI ZHANG, JIAN YIN LI, MING FANG ZHANG and ZHU MING GUO \\ Department of Head and Neck Surgery, Cancer Centre of Sun Yat-sen University, Guangzhou, Guangdong 510060, P.R. China
}

Received April 30, 2016; Accepted April 6, 2017

DOI: $10.3892 / \mathrm{ol} .2017 .7139$

\begin{abstract}
Accurate evaluation of oral tissue defects following oncological surgery is necessary for the subsequent reconstruction. However, there is currently no effective classification system for oral defects in the clinical setting. The present study therefore developed a clinical classification system for the evaluation and reconstruction of oral defects. A retrospective cohort study was performed. A two-dimensional classification system based on coronal computed tomography/magnetic resonance imaging was developed and validated by 145 cases with oral defects. Oral defects could be classified into 6 types (I-VI) horizontally and 2 classes (a and b) vertically. The proportion of the various types was as follows: Type I, $35.9 \%$; type II, $21.4 \%$; type III, $23.4 \%$; type IV, $4.8 \%$; type V, $2.1 \%$; and type VI, $12.4 \%$. Among them, 91 cases $(62.8 \%)$ were class a and 54 cases $(37.2 \%)$ were class b. Type Ia-Va represented the unilateral 1-5 subsites involving superficial oral defects without mandibular continuity destruction (88 cases, 60.7\%). Type $\mathrm{Ib}-\mathrm{Vb}(+\mathrm{M})$ represented the unilateral 1-5 subsites involving deep oral defects with segmental mandibular continuity destruction (38 cases, 26.2\%). Type I-V (+S) represented the unilateral through and through oral defects with cheek skin involvement (10 cases, 6.9\%). Type VI represented bilateral oral defects (18 cases, 12.4\%). The present classification system for the evaluation of the oral defects was simple and practical, and could identify the common types of oral defects and guide the reconstruction.
\end{abstract}

\section{Introduction}

The incidence of oral cancer, particularly squamous cell carcinomas (OSCC), is high in Southern China and there is also a rising trend worldwide $(1,2)$. Surgery serves an important role in the treatment of OSCC (3). Oral defects

Correspondence to: Dr Wei Wei Liu, Department of Head and Neck Surgery, Cancer Centre of Sun Yat-sen University, 651 East Dongfeng Road, Guangzhou, Guangdong 510060, P.R. China

E-mail: liuww@sysucc.org.cn

Key words: oral cancer, tissue defects, reconstruction, classification, flaps following oncological surgery may affect facial appearance and oral function; therefore, reconstruction is an indispensable part of oral cancer surgery (4). Reconstructive methods used include using free flaps, prosthesis or implantable materials. However, regardless of the method used, an accurate evaluation of oral tissue defects is necessary. Anatomically, the oral cavity comprises the upper, lateral and lower walls. The upper wall is mainly bony structures, including the hard palate and superior alveolus. A maxillectomy defect is a defect associated with the upper wall of the oral cavity, which has been well evaluated in the literature (5-8). However, the lateral and lower wall of the oral cavity contains not only mandibular structures, but also soft tissues, including the mobile tongue, the floor of the mouth and the cheeks. Therefore, due to the complexed anatomy and the existence of varied and compound postoperative defects, there is no effective method for the evaluation of oral defects occurring in the lower region of the oral cavity. The establishment of a classification system for postoperative oral defects could therefore aid clinical evaluation and guide oral reconstruction.

There have been few previous studies regarding the clinical evaluation of head and neck defects. For bony defects, there have been several studies delineating the classification for maxillectomy defects that defined the range of a maxillary defect in horizontal and vertical dimensions (5-8). Schultz et al (9) and Boyd et al (10) also established a classification system for mandibular defects (11). For oropharyngeal soft tissue defects following a transoral robotic surgery procedure, de Almeida et al (12) classified them into 4 types and provided the algorithm for the reconstruction. Brown's classification covers the evaluation for the upper wall of the oral cavity $(5,6)$, however, there remains no effective classification system for compound oral defects occurring in the lower region of the oral cavity.

The present study reviewed a group of cases with oral defects occurring in the lower region of the oral cavity and proposed a classification system for the clinical evaluation and oral reconstruction.

\section{Materials and methods}

Patients. Clinical data of 145 patients with oral tumors treated in the Department Of Head And Neck Surgery of Cancer Center of Sun Yat-Sen University (Guangdong, China) between January 2010 and December 2013 were reviewed. Inclusion 
criteria for the present study were as follows: All patients were pathologically diagnosed as having primary oral tumors, mainly SCC; all patients received radical curative surgery; all postoperative oral defects received primary reconstruction; and the clinical data were complete. Oral cancer arising from the lip, hard palate and upper gum, oropharyngeal cancer and cheek skin cancer were excluded from the present study. The site and extent of the tumor were evaluated by clinical examination and computed tomography/magnetic resonance imaging. The postoperative oral defects were evaluated using preoperative imaging and surgical records. The detailed data for the 145 patients with oral tumors are summarized in Table I.

Classification system. The present study established a classification system for the evaluation of postoperative oral defects based on two dimensions. The extent of the oral defect from inside out was evaluated in the horizontal dimension. The extent of the depth of the oral defect was evaluated in the vertical dimension. Two straight lines were then introduced into this classification system. The midline vertically divided the oral cavity into 2 symmetrical parts, and the horizontal line connecting midpoints of the bilateral mandible in the coronary section indicated the level of mandibular nerve divided the oral cavity into 2 regions in terms of depth. The 5 anatomical subsites of the oral cavity were horizontally defined as the ipsilateral hemi-tongue (T), the floor of the mouth $(\mathrm{F})$, the mandible (M), the buccal mucosa (B) and the cheek skin (S). The depth of the oral defects defined by the horizontal line included shallow (a) and deep (b) types. This classification system is represented in Fig. 1.

According to the missing numbers of continuous oral anatomical subsites, oral defects in the horizontal dimension could be classified into 6 types (I-VI). According to the depth, oral defects in the vertical dimension could be classified into 2 types ( $a$ and $b$ ). The detailed definitions are shown in Table II.

According to this classification system, postoperative oral defects can be defined into 12 types (Fig. 1). Each type of tissue defect could be expressed with an alphabetical sign; for example, IIIb (FMB) indicating an IIIb oral defect with 3 continuous subsites (floor of mouth, mandible and buccal mucosa) involved.

\section{Results}

A total of 145 patients with oral tumors received curative radical surgery, and their postoperative oral defects were evaluated using the proposed classification system. The results are displayed in Table III. The proportion of different types of oral defects was as follows: Type I, 35.9\%; type II, 21.4\%; type III, 23.4\%; type IV, 4.8\%; type V, 2.1\%; and type VI, $12.4 \%$. Among them, $91(62.8 \%)$ cases were type a and 54 (37.2\%) cases were type b.

To further elucidate the clinical relevance, the classifications in Table III could be summarized into 4 groups (counting may overlap as some patients were included in more than one category in order to highlight facial skin and bone involvement): Ia-Va representing the unilateral 1-5 subsites involving superficial oral defects without mandibular continuity destruction (88 cases, $60.7 \%)$; $\mathrm{Ib}-\mathrm{Vb}(+\mathrm{M})$ representing the unilateral 1-5 subsites involving deep oral defects
Table I. Clinical features of 145 patients with oral tumors.

\begin{tabular}{|c|c|c|}
\hline Clinical features & Patients, $\mathrm{n}$ & $\%$ \\
\hline \multicolumn{3}{|l|}{ Sex } \\
\hline Male & 101 & 69.66 \\
\hline Female & 44 & 30.34 \\
\hline \multicolumn{3}{|l|}{ Age, years } \\
\hline$<40$ & 32 & 22.07 \\
\hline$>40$ & 113 & 77.93 \\
\hline \multicolumn{3}{|l|}{ Primary sites } \\
\hline Tongue & 69 & 47.59 \\
\hline Floor of mouth & 26 & 17.93 \\
\hline Mandible & 10 & 6.90 \\
\hline Gingiva & 24 & 16.55 \\
\hline Buccal mucosa & 16 & 11.03 \\
\hline \multicolumn{3}{|l|}{ Pathology } \\
\hline Squamous cell carcinoma & 125 & 86.21 \\
\hline Others & 20 & 13.79 \\
\hline \multicolumn{3}{|l|}{$\mathrm{T}^{\mathrm{a}}$} \\
\hline 1 & 8 & 6.40 \\
\hline 2 & 34 & 27.20 \\
\hline 3 & 51 & 40.80 \\
\hline 4 & 32 & 25.60 \\
\hline \multicolumn{3}{|l|}{$\mathrm{N}^{\mathrm{a}}$} \\
\hline 0 & 50 & 40.00 \\
\hline 1 & 38 & 30.40 \\
\hline 2 & 34 & 27.20 \\
\hline 3 & 3 & 2.40 \\
\hline \multicolumn{3}{|l|}{ Treatment } \\
\hline Surgery alone & 76 & 52.41 \\
\hline Surgery+RT & 22 & 15.17 \\
\hline ICT+surgery & 23 & 15.86 \\
\hline Other therapies with surgery & 24 & 16.55 \\
\hline
\end{tabular}

${ }^{a}$ Only patients with squamous cell carcinoma were staged $(n=125) . T$, primary $\mathrm{T}$ stage; $\mathrm{N}$, cervical $\mathrm{N}$ stage.

with segmental mandibular continuity destruction (39 cases, $26.9 \%)$; I-V (+S) representing the unilateral through and through oral defects with cheek skin involvement (7 cases, $4.8 \%)$; and VI representing bilateral oral defects (18 cases, $12.4 \%)$

The different types of representative postoperative oral defects are shown in representative in Fig. 2. All cases received primary surgical reconstruction and Table IV demonstrates the reconstructive methods used in this series of patients. A total of 4 reconstructive methods were used in 145 cases: 101 cases $(69.7 \%)$ with free flaps; 21 cases $(14.5 \%)$ with flaps combined with titanium plate reconstruction; 13 cases $(9.0 \%)$ with local pedicled flaps; and 10 cases (6.9\%) with other methods. Among the 101 cases which received free flap reconstruction: 79 cases (78.2\%) used an anterolateral thigh flap; 5 cases (5.0\%) used a forearm flap; and 17 cases (16.8\%) used a fibular flap. Among the 21 cases reconstructed by flaps combined with titanium 


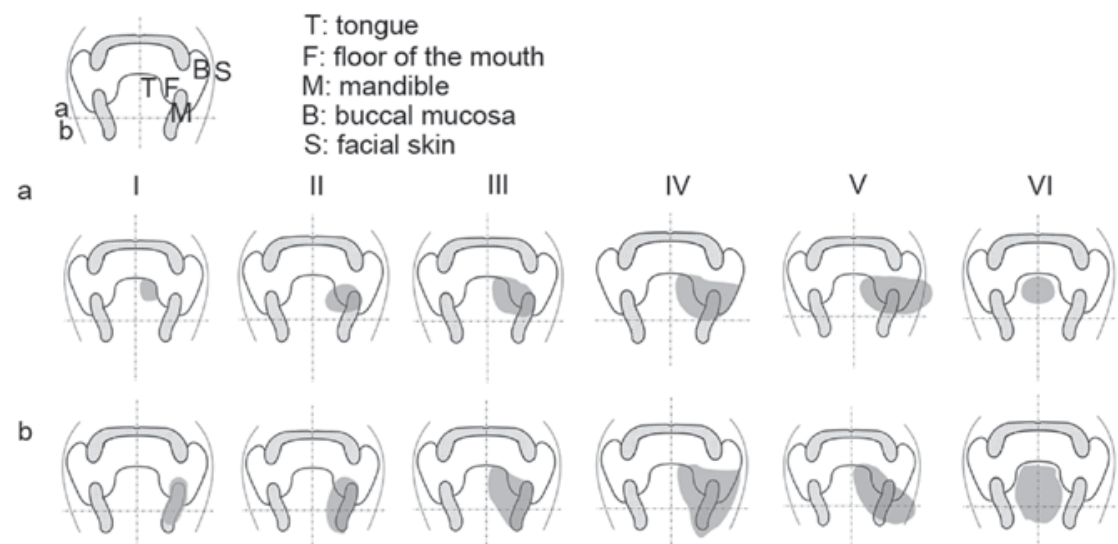

Figure 1. Representation of 12 types of oral defects following oncological surgery. Stages I-VI were used to classify the horizontal extent of the oral defect, whilst ( $a$ and $b$ ) were used to classify the vertical extent of the defect. $b$, represents shallow oral defects and b, represent deep oral defects.

plates, 16 cases used free anterolateral thigh flaps and 5 cases used pectoralis major myocutaneous flaps. Other reconstructive methods included adjacent tissue flaps (such as tongue flaps, forehead flaps and strap muscle flaps) and a free skin graft.

\section{Discussion}

An ideal reconstruction requires the accurate evaluation of the tissue defects. An effective clinical classification system should distinguish between simple and complicated defects, clearly express the characteristics of the defects, and be used easily and practically. The oral cavity is the entrance of the digestive tract and can anatomically be abstracted as a hollow tubular structure, delineated as a circle in Fig. 1. As defects of the upper jaw could be included in maxillary defects, the evaluation of oral defects was mainly concentrated on the lower half circle that was the basal and lateral walls. Therefore, it was considered that the extent of the oral defects could be sufficiently evaluated in two dimensions. Horizontally, it was possible to measure the area of the defect from inside out by the anatomical subsites involved and whether the defect was through facial skin or not; vertically, the depth of the oral defect was measured, and it could be determined whether the mandibular continuity was destroyed or not. The current classification system for the evaluation of postoperative oral defects, except from the upper jaw, was based on the above concepts.

Oral defects following oncological surgery for tumors are complicated. Therefore, two parameters were used to summarize the complexity of oral defects in the current classification system: The numbers of continuous oral anatomical subsites involved in the defect and the depth of the oral defects. The former could not only reflect the area of the defect, but also clarify complicated defects; for example, a through and through defect or a defect across the midline. The latter could express the depth of the defect, particularly the mandibular continuity. Therefore, the more anatomical subsites that were involved and the deeper the defect was, the more complicated the defect was. Accordingly, in the present classification system, higher level and b-type defects represented more complicated defects. In the present study, among the 145 cases with the oral defects, 28 cases (19.3\%) were classified as type IV-VI, which were difficult defects that required more complicated reconstruction.

The postoperative oral defects observed in the present study were varied and the classification system should therefore be able to reflect the special types of oral defects. As different reconstructive strategy may be required in certain oral defects, the following 3 conditions should receive additional attention: Through and through defects; composite defects combined with mandibular discontinuity; and bilateral oral defects. In the present classification system, the above 3 types of special oral defects have been clearly indicated. Through and through oral defects could be evaluated by cheek skin involvement $(+S)$. Composite oral defects with mandibular involvement could be expressed as $(+\mathrm{M})$, which could then be further classified into type a (without mandibular continuity destruction) and type $b$ (with mandibular continuity destruction). Type VI could reflect the bilateral oral defects.

The present proposed classification system for oral defects is concise and should be easy to use clinically. The 2 parameters used in the classification system are familiar to surgeons and could be easily evaluated on preoperative coronary images of the oral cavity. In addition, it is easy to record types of oral defects using this classification system. The present classification could describe a through and through oral defect with the involvement of 4 subsites (floor of mouth, mandible, buccal mucosa and facial skin) and in combination with mandibular continuity destruction simply by designating it as type IVb (MBS).

In order to evaluate the depth of the oral defects and determine whether mandibular continuity was destroyed, a horizontal line was introduced into the present classification system. Rectangular and segmental mandibulectomy were the two main surgical procedures used in the treatment of OSCC with mandibular invasion. This horizontal line could be used to distinguish the two conditions, as anatomically, this line was almost on the same level as the mandibular nerve, and by discriminating shallow from deep oral defects this line may guide the choice of a different reconstructive method.

There were no parameters to evaluate the extent of oral defects in the sagittal dimension in the present proposed 
Table II. Definitions for the classification system of oral defects.

\section{A, Horizontal}

\begin{tabular}{|c|c|c|}
\hline Types & Subsites & Definition \\
\hline \multirow[t]{5}{*}{ I } & & $\begin{array}{l}\text { Defects with only } 1 \text { ipsilateral } \\
\text { subsite involved }\end{array}$ \\
\hline & $\mathrm{T}$ & Tongue \\
\hline & $\mathrm{F}$ & Floor of mouth \\
\hline & M & Mandible \\
\hline & $\mathrm{B}$ & Buccal mucosa \\
\hline \multirow[t]{5}{*}{ II } & & $\begin{array}{l}\text { Defects with } 2 \text { ipsilateral } \\
\text { continuous subsites involved }\end{array}$ \\
\hline & $\mathrm{TF}$ & Tongue and floor of mouth \\
\hline & FM & Floor of mouth and mandible \\
\hline & MB & Mandible and buccal mucosa \\
\hline & $\mathrm{BS}$ & Buccal mucosa and facial skin \\
\hline \multirow[t]{4}{*}{ III } & & $\begin{array}{l}\text { Defects with } 3 \text { ipsilateral } \\
\text { continuous subsites involved }\end{array}$ \\
\hline & TFM & $\begin{array}{l}\text { Tongue, floor of mouth and } \\
\text { mandible }\end{array}$ \\
\hline & FMB & $\begin{array}{l}\text { Floor of mouth, mandible and } \\
\text { buccal mucosa }\end{array}$ \\
\hline & MBS & $\begin{array}{l}\text { Mandible, buccal mucosa and } \\
\text { facial skin }\end{array}$ \\
\hline \multirow[t]{3}{*}{ IV } & & $\begin{array}{l}\text { Defects with } 4 \text { ipsilateral } \\
\text { continuous subsites involved }\end{array}$ \\
\hline & TFMB & $\begin{array}{l}\text { Tongue, floor of mouth, mandible } \\
\text { and buccal mucosa }\end{array}$ \\
\hline & FMBS & $\begin{array}{l}\text { Floor of mouth, mandible, buccal } \\
\text { mucosa and facial skin }\end{array}$ \\
\hline $\mathrm{V}$ & TFMBS & $\begin{array}{l}\text { Defects with all of the } 5 \text { ipsilateral } \\
\text { subsites involved }\end{array}$ \\
\hline VI & & $\begin{array}{l}\text { Defects with bilateral subsites } \\
\text { involved }\end{array}$ \\
\hline
\end{tabular}

B, Vertical

$\begin{array}{ll}\mathrm{a} & \text { Defects above the horizontal line } \\ \mathrm{b} & \text { Defects across the horizontal line } \\ \text { to include deep floor of mouth } \\ \text { and whole height of mandible }\end{array}$

classification system, as in the case of oral cancer with posterior invasion of the tongue base or tonsil, the oropharyngeal defect could be evaluated by de Almeida's system (12). In addition, the oral defects involving the oropharynx would not significantly alter the method of reconstruction.

The establishment of the present classification system could aid the measurement of the extent of the oral defects, distinguish special types of defects and guide the choice of reconstruction. Previous studies on the classification of defects of the upper jaw, mandible, mid-face and oropharynx provided
Table III. Postoperative classification of defects for 145 patients with oral tumors.

\begin{tabular}{llccc}
\hline Types & Subsites & Type a & Type b & Total, $\mathrm{n}(\%)$ \\
\hline I & T & 31 & - & 31 \\
& F & 5 & - & 5 \\
& M & 2 & 5 & 7 \\
& B & 9 & - & 9 \\
& & 47 & 5 & $52(35.9)$ \\
II & TF & 8 & 1 & 9 \\
& FM & 10 & 7 & 17 \\
& MB & 4 & - & 4 \\
& BS & 1 & - & 1 \\
III & & 23 & 8 & $31(21.4)$ \\
& TFM & 15 & 14 & 29 \\
& FMB & - & 3 & 3 \\
& MBS & 2 & - & 2 \\
IV & & 17 & 17 & $34(23.4)$ \\
& TFMB & - & 3 & 3 \\
& FMBS & 1 & 3 & 4 \\
V & & 1 & 6 & $7(4.8)$ \\
& & - & 3 & $3(2.1)$ \\
& & 3 & 15 & $18(12.4)$ \\
\hline
\end{tabular}

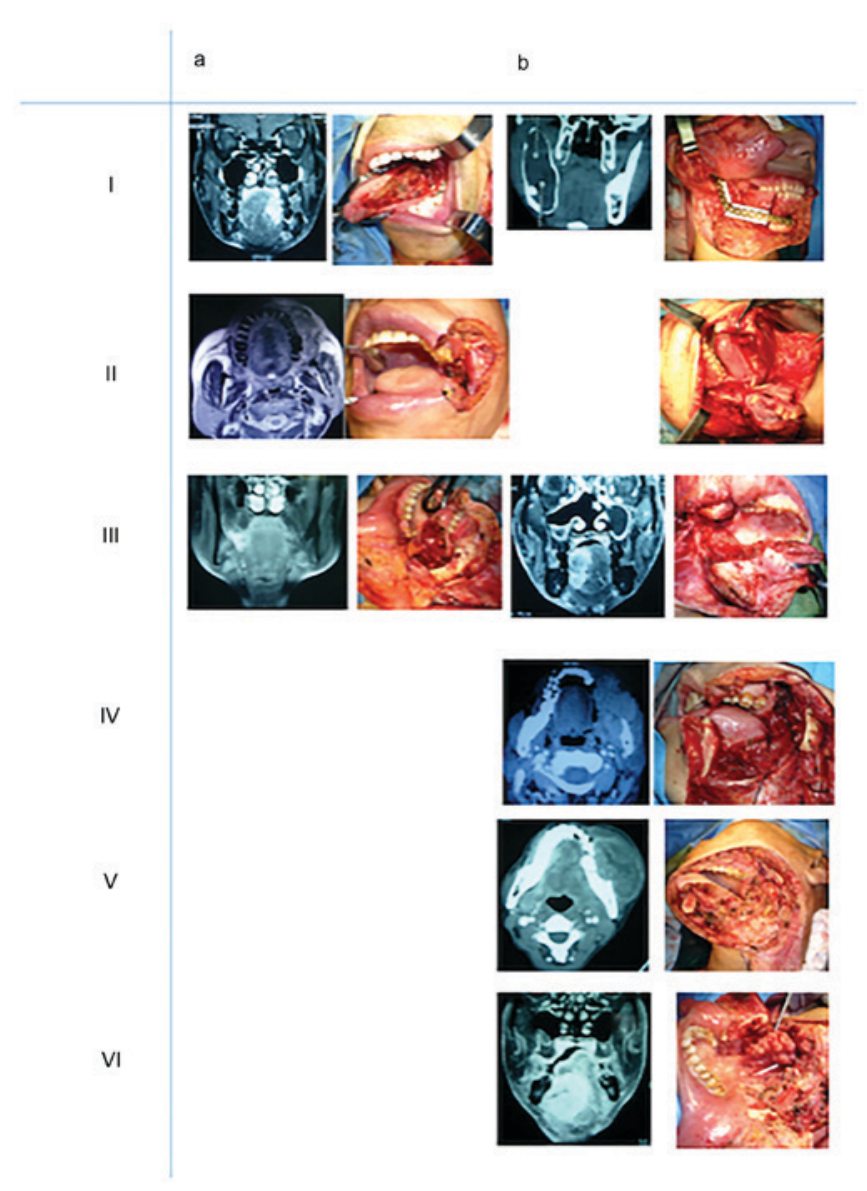

Figure 2. Representative cases of patients with each type of oral defects. Stages I-VI were used to classify the horizontal extent of the oral defect, whilst ( $a$ and $b$ ) were used to classify the vertical extent of the defect. $\mathrm{a}$, represents shallow oral defects and $\mathrm{b}$, represent deep oral defects. 
Table IV. Reconstructive methods used for all types of oral defects.

\begin{tabular}{|c|c|c|c|c|c|c|c|}
\hline \multirow[b]{2}{*}{ Types } & \multicolumn{3}{|c|}{ Free flap } & \multicolumn{2}{|c|}{ Flap+titanium plate } & \multirow{2}{*}{$\begin{array}{c}\text { Local flap } \\
\text { PM }\end{array}$} & \multirow[b]{2}{*}{ Others } \\
\hline & ALT & $\mathrm{FF}$ & $\mathrm{RF}$ & $\mathrm{ALT}+\mathrm{T}$ & $\mathrm{PM}+\mathrm{T}$ & & \\
\hline Ia & 32 & - & 4 & - & - & 1 & 9 \\
\hline $\mathrm{Ib}$ & - & 6 & - & - & - & - & - \\
\hline IIa & 16 & - & 1 & 2 & 1 & 1 & - \\
\hline $\mathrm{IIb}$ & 2 & 4 & - & - & 2 & 2 & - \\
\hline IIIa & 10 & - & - & 1 & - & 5 & 1 \\
\hline IIIb & 4 & 2 & - & 7 & 1 & 3 & - \\
\hline IVa & - & - & - & - & - & - & - \\
\hline $\mathrm{IVb}$ & 1 & 2 & - & 3 & 1 & - & - \\
\hline $\mathrm{Va}$ & - & - & - & - & - & - & - \\
\hline $\mathrm{Vb}$ & 1 & - & - & 2 & - & - & - \\
\hline VIa & 3 & - & - & - & - & - & - \\
\hline $\mathrm{VIb}$ & 10 & 3 & - & 1 & - & 1 & - \\
\hline
\end{tabular}

ALT, anterolateral thigh flap; FF, fibular flap; RF, radial forearm flap; T, titanium plate; PM, pectoralis major flap.

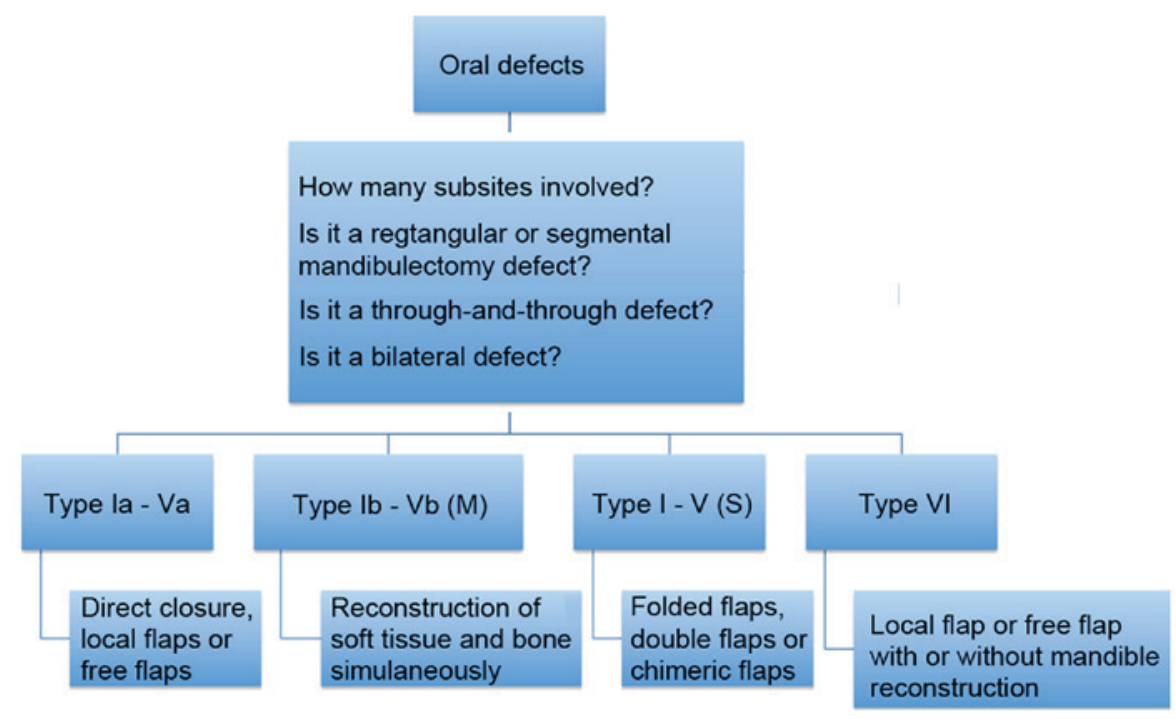

Figure 3. Multiple factors including the numbers of involved subsites, special missing tissue like bone or facial skin and bilateral defects were evaluated to produce 4 groups of oral defects were classified and their relevant reconstructive methods were provided. Stages I-VI were used to classify the horizontal extent of the oral defect, whilst ( $a$ and $b$ ) were used to classify the vertical extent of the defect. a, represents shallow oral defects and b, represent deep oral defects. $\mathrm{M}$, mandible; S, facial skin.

an algorithm for reconstruction (5-12). As an example, the classification of upper jaw defects evaluated the extent using only two dimensions, the advantage being that different defects were easily identifiable in the clinical setting $(5,6)$. For minor defects such as class $1 \mathrm{a}, 2 \mathrm{a}$ or $2 \mathrm{~b}$, an obturator may be chosen in the reconstruction. Free flaps would be the better choice for large defects like class $4 \mathrm{a}$ or $4 \mathrm{~b}$. We also saw the pattern in the oral defects just as in the midfacial defects and organized them into clinically actionable groups. The present classification system could summarize the 4 common types of oral defects and guide the choice of reconstructive methods (Fig. 3). For stage Ia-Va oral defects, due to a shallow loss of tissue, the absence of destruction to mandibular continuity and the complexity of the defects decided only by numbers of involved anatomical subsites, reconstruction could be completed by simple closure, local flaps or free flaps according to the area of missing tissues. In this type of oral defect, as the continuity of the mandible was preserved, it was relatively easy to add dental implants. The restorability of oral function in these types of defects should be good.

For type b (+M) defects with segmental mandibulectomy, free fibula flaps or free flaps combined with titanium plates should be considered for the reconstruction. The size of the defect should be evaluated, as this may affect the choice of the flaps and reconstructive plates. Occasionally, three-dimensional computer-aided design was required to restore the 
anatomy for certain bulky or wide compound oral defects. Dental implants were feasible for these types of defects, however, the restorability of the oral function depended not only on the size of the defect itself, but also on the expected oncological result. For the through and through defects with facial skin involvement, folding flaps, double flaps or chimeric flaps may be the most suitable reconstruction choice. The oral function could be restored well for these types of defects. For bilateral oral defects, a large block of composite tissue transfer was generally required to reconstruct the total tongue, the floor of the mouth and a region of the mandible. Due to a large volume of missing oral tissue, the restorability of the oral function in this type of defect may be compromised. If a total glossectomy was performed, the swallowing function may also be affected.

There were numerous factors associated with the evaluation of postoperative oral defects. The present classification system was based on the coronary anatomy and introduced 2 parameters, including the number of involved anatomical subsites and the depth of the defect, to evaluate mandibular continuity and distinguish special oral defects as bilateral or through and through defects. This classification was only a two-dimensional system, not including the sagittal dimension, which influenced the complete evaluation of the oral defects to a certain extent. In addition, it is necessary to evaluate additional cases of oral defects using the proposed classification system to further validate its efficacy and practicability. Moreover, this classification could not provide an evaluation for the size of the harvested flap. Another limitation of the present classification system was that it could not define the specific site of a mandibular defect when a compound defect appeared, and it could not differentiate between anterior and posterior mandibular defects that may influence the choice of different reconstructive methods. Further modifications are therefore required in order to improve the classification system. Other associated factors, including adjuvant radiotherapy and the systemic health of the patient, should also be considered in the development of the classification system.
In summary, an accurate evaluation of oral defects is necessary to guide the subsequent reconstruction. The present retrospective study proposed a novel classification system for the evaluation of oral defects, and confirmed that it was simple and clinically practical. In the future, this classification system could identify the common types of oral defects and assist in guiding the reconstruction.

\section{References}

1. Saman DM: A review of the epidemiology of oral and pharyngeal carcinoma: Update. Head Neck Oncol 4: 1, 2012.

2. Chaturvedi AK, Anderson WF, Lortet-Tieulent J, Curado MP, Ferlay J, Franceschi S, Rosenberg PS, Bray F and Gillison ML: Worldwide trends in incidence rates for oral cavity and oropharyngeal cancers. J Clin Oncol 31: 4550-4559, 2013.

3. OW TJ and Myers JN: Current management of advanced resectable oral cavity squamous cell carcinoma. Clin Exp Otorhinolaryngol 4: 1-10, 2011.

4. Wong CH and Wei FC: Microsurgical free flap in head and neck reconstruction. Head Neck 32: 1236-1245, 2010.

5. Brown JS, Rogers SN, McNally DN and Boyle M: A modified classification for the maxillectomy defect. Head Neck 22: 17-26, 2000.

6. Brown JS and Shaw RJ: Reconstruction of the maxilla and midface: Introducing a new classification. Lancet Oncol 11: 1001-1008, 2010.

7. Cordeiro PG and Santamaria E: A classification system and algorithm for reconstruction of maxillectomy and midfacial defects. Plast Reconstr Surg 105: 2331-2348, 2000.

8. Bidra AS, Jacob RF and Taylor TD: Classification of maxillectomy defects: A systematic review and criteria necessary for a universal description. J Prosthet Dent 107: 261-270, 2012.

9. Schultz BD, Sosin M, Nam A, Mohan R, Zhang P, Khalifian S, Vranis N, Manson PN, Bojovic B and Rodriguez ED: Classification of mandible defects and algorithm for microvascular reconstruction. Plast Reconstr Surg 135: 743e-754e, 2015.

10. Boyd JB, Gullane PJ, Rotstein LE, Brown DH and Irish JC: Classification of mandibular defects. Plast Reconstr Surg 92: 1266-1275, 1993.

11. Brown JS, Barry C, Ho M and Shaw R: A new classification for mandibular defects after oncological resection. Lancet Oncol 17: e23-e30, 2016.

12. de Almeida JR, Park RC, Villanueva NL, Miles BA, Teng MS and Genden EM: Reconstructive algorithm and classification system for transoral oropharyngeal defects. Head Neck 36: 934-941, 2014. 